\title{
Subjective phenomena elicited by intracranial electrical stimulation challenge a global neuronal workspace hypothesis: A reply to objections raised by Naccache et al.
}

\author{
${ }^{*}$ Omri Raccah ${ }^{1}$, Ned Block ${ }^{2}$, \& Kieran C.R. Fox ${ }^{3,4}$ \\ ${ }^{1}$ Department of Psychology, New York University, \\ ${ }^{2}$ Department of Philosophy, New York University, \\ ${ }^{3}$ Department of Neurology \& Neurological Sciences, Stanford University, \\ ${ }^{4}$ School of Medicine, Stanford University
}

* Correspondence to: or409@nyu.edu

In their commentary (Naccache et al., 2021) on our paper (Raccah et al., 2021), Naccache et al.'s main claim is that the complex and distributed functional organization of the prefrontal cortex (PFC) - relative to sensory cortices - precludes its functional modulation by local intracranial electrical stimulation (iES). Specifically, the authors expect that iES to PFC subregions will produce "a random pattern of activity that does not correspond to any meaningful mental content when applied to PFC." Naccache et al. note that we raise the issue of distributed representation ourselves, but they believe we fail to acknowledge the consequences: "What [Raccah et al.] fail to discuss is that the activation of large vectors of activity, distributed over many neurons, may be needed to elicit a meaningful PFC content." Instead, Naccache et al. suggest that electrical microstimulation or optogenetic techniques, in combination with single-unit recordings "should be capable of inducing a predetermined conscious content, better than gross iES."

Our reply both in our original paper (Raccah et al., 2021, p. 2078) and now is that, if global neuronal workspace theory (GNWT) or higher order theories are correct, applying iES to PFC should reliably perturb ongoing perception, e.g. of the doctor's face or what the doctor is saying but it does not. Naccache et al. are probably correct that iES is too crude to create a meaningful percept ex nihilo, but it is not too crude to perturb an ongoing percept. While it is true that subjective effects in the PFC are especially rare following stimulation relative to the rest of the cortex (Fox et al., 2020), the argument posed by Naccache et al. is simply contradicted by many examples in which local iES to PFC regions produced robust and reproducible effects - except that these effects were not perceptual in nature. In addition to many consistent effects in the orbiotofrontal cortex (OFC) and anterior cingulate cortex (ACC), reproducible effects have been reported in the dorso- and ventro-lateral PFC (Liu et al., 2020; Popa et al., 2016), but these reports were of abstract conceptual thought (consistent with functional neuroimaging evidence; Berkovich-Ohana et al., 2020), rather than perturbations of conscious perception. Notably, this also includes a remarkable variety of motor effects found across PFC subregions (Selimbeyoglu \& Parvizi, 2010).

Naccache et al. point to the effects of iES in the ACC and OFC, as predicted by the global neuronal workspace theory. However, the authors give no explanation as to why, if ACC and OFC are part of a general consciousness circuit, stimulation to them does not produce perturbations in visual or auditory experience across dozens of individual cases (Fox et al., 2018; Raccah et al., 2021; Rao et al., 2018; Yih et al., 2019). The GNWT should be able to offer an explanation as to why stimulation to the ACC and OFC produces such a specific group of effects - somatic, visceral, emotional, olfactory - instead of perturbations of ongoing perceptual representations. Critically, these effects are consistent with the known functional roles supported by these regions (Bush et al., 2000; Devinsky et al., 1995; Rolls, 2004). These specialized and consistent iES effects, which are always entirely unrelated to ongoing perceptual content, more likely suggest a link between these local sites and particular conscious content than a constitutive role in consciousness (Malach, 2021).

Naccache et al. note that iES can induce changes in neuronal excitability beyond the stimulation site and suggest that evidence presented in our work is limited by a lack of whole- 
brain sampling methods: "...while the results compiled by Raccah et al. are of primary importance, the absence of simultaneous recordings of global brain activity elicited by iES is a key limitation of current methods." We agree with Naccache et al. that such methods are critical for inferring regional contributions following stimulation, and will be useful for interpreting iES effects outside the PFC. One could also consider using evoked effective connectivity methods for this purpose (Keller et al., 2014). However, this point is in contradiction with Naccache et al.'s criticism that iES is too crude to elicit effects in the PFC. In our view, the fact that iES has widespread effects, including widespread effects within PFC areas, makes it even more remarkable that there are no reports of changes in ongoing perceptual experience following stimulation to PFC regions.

Notably, Baars and colleagues, who also responded to our work (Baars et al., 2021), state that on their version of global workspace theory (Baars et al., 2013), PFC is not crucial to conscious perception. We identified our targets as including the global neuronal workspace theory (Mashour et al., 2020), a theory that does commit to PFC representation. Naccache et al. are advocates of that theory and one can see from their reply that they retain their commitment to a constitutive role for the PFC in conscious perception.

Understanding the neural basis of human consciousness is perhaps the most difficult challenge in all of science, and we welcome diverse perspectives in this debate. We hope that our synthesis continues to provoke lively discussion and that it might stimulate further research in this domain. 


\section{References}

Baars, B. J., Franklin, S., \& Ramsøy, T. Z. (2013). Global workspace dynamics: cortical "binding and propagation" enables conscious contents. Frontiers in psychology, 4, 200.

Baars, B. J., Kozma, R., \& Geld, N. (2021). Global workspace theory (GWT) and prefrontal cortex: A reply to Raccah et al. Journal of Neuroscience eLetters.

Berkovich-Ohana, A., Noy, N., Harel, M., Furman-Haran, E., Arieli, A., \& Malach, R. (2020). Inter-participant consistency of language-processing networks during abstract thoughts. Neuroimage, 211, 116626.

Bush, G., Luu, P., \& Posner, M. I. (2000). Cognitive and emotional influences in anterior cingulate cortex. Trends in cognitive sciences, 4(6), 215-222.

Devinsky, O., Morrell, M. J., \& Vogt, B. A. (1995). Contributions of anterior cingulate cortex to behaviour. Brain, $118(1), 279-306$.

Fox, K. C., Shi, L., Baek, S., Raccah, O., Foster, B. L., Saha, S., Margulies, D. S., Kucyi, A., \& Parvizi, J. (2020). Intrinsic network architecture predicts the effects elicited by intracranial electrical stimulation of the human brain. Nature human behaviour, 1-14.

Fox, K. C., Yih, J., Raccah, O., Pendekanti, S. L., Limbach, L. E., Maydan, D. D., \& Parvizi, J. (2018). Changes in subjective experience elicited by direct stimulation of the human orbitofrontal cortex. Neurology, 91(16), e1519-e1527.

Keller, C. J., Honey, C. J., Entz, L., Bickel, S., Groppe, D. M., Toth, E., Ulbert, I., Lado, F. A., \& Mehta, A. D. (2014). Corticocortical evoked potentials reveal projectors and integrators in human brain networks. Journal of Neuroscience, 34(27), 9152-9163.

Liu, A., Friedman, D., Barron, D. S., Wang, X., Thesen, T., \& Dugan, P. (2020). Forced conceptual thought induced by electrical stimulation of the left prefrontal gyrus involves widespread neural networks. Epilepsy \& Behavior, 104, 106644.

Malach, R. (2021). Local neuronal relational structures underlying the contents of human conscious experience. Neuroscience of Consciousness, 2021(2), niab028.

Mashour, G. A., Roelfsema, P., Changeux, J.-P., \& Dehaene, S. (2020). Conscious processing and the global neuronal workspace hypothesis. Neuron, 105(5), 776-798.

Naccache, L., Changeux, J.-P., Panagiotaropoulos, T. I., \& Dehaene, S. (2021). Why intracranial electrical stimulation of the human brain suggests an essential role for prefrontal cortex in conscious processing: a commentary on Raccah et al. OSF Preprints.

Naccache, L., Changeux, J.-P., Panagiotaropoulos, T. I., \& Dehaene, S. (2021). Why intracranial electrical stimulation of the human brain suggests an essential role for prefrontal cortex in conscious processing: a commentary on Raccah et al. Journal of Neuroscience eLetters.

Popa, I., Donos, C., Barborica, A., Opris, I., Mălîia, M. D., Ene, M., Ciurea, J., \& Mîndruţă, I. (2016). Intrusive thoughts elicited by direct electrical stimulation during stereo-electroencephalography. Frontiers in neurology, 7, 114.

Raccah, O., Block, N., \& Fox, K. C. (2021). Does the prefrontal cortex play an essential role in consciousness? Insights from intracranial electrical stimulation of the human brain. Journal of Neuroscience, 41(10), 20762087.

Rao, V. R., Sellers, K. K., Wallace, D. L., Lee, M. B., Bijanzadeh, M., Sani, O. G., Yang, Y., Shanechi, M. M., Dawes, H. E., \& Chang, E. F. (2018, Dec 17). Direct Electrical Stimulation of Lateral Orbitofrontal Cortex Acutely Improves Mood in Individuals with Symptoms of Depression. Curr Biol, 28(24), 3893-3902.e3894.

Rolls, E. T. (2004). The functions of the orbitofrontal cortex. Brain and cognition, 55(1), 11-29.

Selimbeyoglu, A., \& Parvizi, J. (2010). Electrical stimulation of the human brain: perceptual and behavioral phenomena reported in the old and new literature. Front Hum Neurosci, 4, 46.

Yih, J., Beam, D. E., Fox, K. C., \& Parvizi, J. (2019). Intensity of affective experience is modulated by magnitude of intracranial electrical stimulation in human orbitofrontal, cingulate and insular cortices. Social cognitive and affective neuroscience, 14(4), 339-351. 